Review Article

\title{
A Comprehensive Review of US FDA-Approved Immune Checkpoint Inhibitors in Urothelial Carcinoma
}

\author{
Fu-Shun Hsu, ${ }^{1,2}$ Chun-Hung Su, ${ }^{3}$ and Kou-How Huang ${ }^{4}$ \\ ${ }^{1}$ Department of Urology, New Taipei City Hospital, New Taipei City, Taiwan \\ ${ }^{2}$ Graduate Institute of Clinical Medicine, National Taiwan University College of Medicine, No. 7 Chung San South Road, \\ Taipei 10002, Taiwan \\ ${ }^{3}$ Genomics Research Center, Academia Sinica, No. 128, Academia Rd., Sec. 2, Taipei 11529, Taiwan \\ ${ }^{4}$ Department of Urology, National Taiwan University Hospital, No. 1, Jen-Ai Rd., Sec. 1, Taipei 10051, Taiwan \\ Correspondence should be addressed to Kou-How Huang; khhuang123@ntu.edu.tw
}

Received 17 August 2017; Accepted 31 October 2017; Published 10 December 2017

Academic Editor: Eyad Elkord

Copyright (C) $2017 \mathrm{Fu}$-Shun Hsu et al. This is an open access article distributed under the Creative Commons Attribution License, which permits unrestricted use, distribution, and reproduction in any medium, provided the original work is properly cited.

\begin{abstract}
Few effective treatment options are available for patients with advanced or metastatic urothelial carcinoma (UC) after unsuccessful first-line platinum-based chemotherapy. To date, immune checkpoint inhibitors are novel therapeutic agents for UC treatment. From May 2016 to May 2017, five anti-PD-1/PD-L1 monoclonal antibodies received accelerated or regular approval from the US Food and Drug Administration (FDA) for the treatment of patients with locally advanced or metastatic UC. The present comprehensive review presents the background information of these five US FDA-approved anticancer agents to provide a basic but concise understanding of these agents for advanced studies. We summarize their immune checkpoint mechanisms, clinical efficacy, recommended usage protocols, adverse events, and the limitations of the PD-L1 biomarker assays.
\end{abstract}

\section{Introduction}

Urothelial carcinoma (UC) is one of the top ten leading causes of cancer death worldwide. UC tumorigenesis is thought to be associated with environmental carcinogenic exposure such as cigarette smoking and chemical exposure [1]. The pathological sites of UC include the renal pelvis and ureter in the upper tract as well as bladder and urethra in the lower tract. Among them, the bladder is the most common site of UC occurrence. In the United States, it was estimated that 79,030 new cases and 16,870 deaths were due to bladder UC in 2017 [2].

Bacillus Calmette-Guérin (BCG), an attenuated live strain of Mycobacterium bovis, has been used for treatment in patients with nonmuscle invasive bladder UC since the 1990s. The benefits from intravesical BCG instillation have been proven, including lowering the risk of disease recurrence and disease progression [3]. BCG is the standard treatment for patients with nonmuscle invasive bladder UC following transurethral resection of bladder tumors for decades, but underlying mechanism of its antitumor effect remains unclear. BCG induces a local inflammatory response and recruits immune cells to destroy tumor cells and, therefore, plays a vital role in bladder cancer immunotherapy. There are some limitations in BCG treatment, including high failure rate and risk of systemic infection.

Cisplatin-based systemic chemotherapy remains the mainstay of treatment in patients with metastatic UC [4]. There are still $30 \%$ to $50 \%$ of advanced UC cases that are not responsive to cisplatin-based chemotherapy. Although some new chemotherapy regimens have been developed, the prognosis for patients with metastatic UC remains poor [5]. Other limiting factors associated with standard regimen are the substantial toxicity and patients' physical conditions [6]. Treatment-related deaths occurred in $2 \%$ to $4 \%$ of patients, especially in the elderly $[4,7]$. The median overall survival (OS) of patients with metastatic UC who received first-line platinum-based chemotherapy ranges from 12 to 15 months, and only approximately $5 \%$ of these patients have a 5-year survival [8-10]. The systemic salvage therapy for 


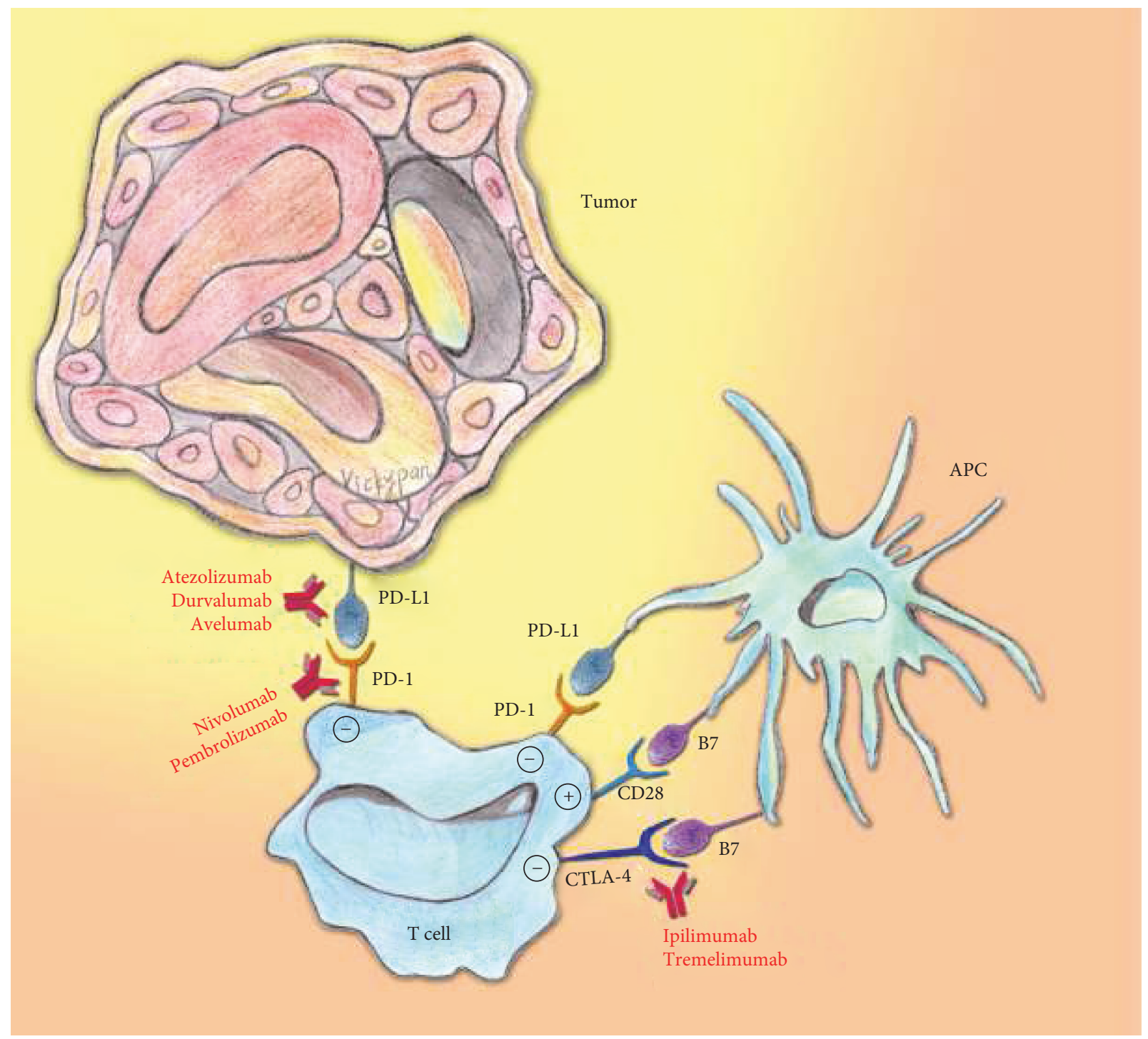

FIGURE 1: Illustration of anticheckpoint immunotherapy. The immune system is maintained and functions in homeostasis. Once CD28 binds to its ligand, B7, on the surface of antigen-presenting cells (APCs), T cell proliferation is activated to enhance immunity. On the other hand, cytotoxic T lymphocyte antigen-4 (CTLA-4) and programmed death-1 (PD-1) on APCs or tumor cells transmit inhibitory signals, while binding to their ligands, B7 and PD-L1, respectively. In general, the immune cells could recognize tumor cells and then destroy them. However, the tumor cells can escape from the host antitumor activities by suppressing the activation of immune cells. The anticheckpoint antibodies are developed to block the inhibitory pathways and then restore $\mathrm{T}$ cell immunity against tumors.

patients with advanced UC lasts only 6 to 8 months [11]. Unlike patients with other cancers, such as non-small-cell lung cancer (NSCLC) [12-14], breast cancer [15, 16], and leukemia [17], who can benefit from many targeted agents, including small molecule inhibitors or anticancer antibodies, patients with UC are still awaiting effective targeted drug treatments. Therefore, there is an urgent need to develop a novel therapy to improve therapeutic efficacy and patient survival or to reduce side effects for patients with locally advanced or metastatic UC.

\section{Immune Checkpoint Therapy}

The immune system defends the body from an invasion by foreign etiological agents. The presentation of antigens to $\mathrm{T}$ cells by antigen-presenting cells (APCs) is a critical process (Figure 1). Several protein molecules involved in the regulation of immune processes and for homeostatic maintenance of the immune system have been identified. CD28 was the first protein to be identified as a coreceptor that transmits stimulatory signals to T cells. After CD28 binds to its ligand, the B7 protein, on the surface of APCs, T cell proliferation is activated to enhance immunity (Figure 1). Cytotoxic T lymphocyte antigen-4 (CTLA-4) and programmed death-1 (PD-1) transmit inhibitory signals when bound to their ligands, B7-1/B72 and PD-L1 (B7-H1)/PD-L2 (B7-DC), respectively, on APCs or tumors (Figure 1). Such protein molecules involved in immune regulation are referred to as immune checkpoints. Typically, the immune system is capable of recognizing and destroying tumor cells; however, tumor cells can exploit the 
TABLE 1: Background information on US FDA-approved PD-1/PD-L1 inhibitors for the treatment of urothelial carcinoma.

\begin{tabular}{|c|c|c|c|c|c|c|c|}
\hline Target & Generic name & Antibody class & $\begin{array}{l}\text { Trade } \\
\text { name }\end{array}$ & Development name(s) & Company & $\begin{array}{l}\text { Recommended dose } \\
\text { and schedule }\end{array}$ & $\begin{array}{c}\text { Date of } \\
\text { approval }\end{array}$ \\
\hline \multirow{2}{*}{ PD-1 } & Nivolumab & Human IgG4 & Opdivo & $\begin{array}{c}\text { BMS-936558, MDX- } \\
\text { 1106, ONO-4538 }\end{array}$ & $\begin{array}{l}\text { Bristol-Myers } \\
\text { Squibb Co. }\end{array}$ & $\begin{array}{c}240 \mathrm{mg} \text {, every } \\
2 \text { weeks }\end{array}$ & 2 Feb. 2017 \\
\hline & Pembrolizumab & Humanized IgG4 & Keytruda & $\begin{array}{c}\text { MK-3475, } \\
\text { lambrolizumab }\end{array}$ & $\begin{array}{l}\text { Merck and } \\
\text { Co. Inc. }\end{array}$ & $\begin{array}{l}200 \text { mg, every } \\
3 \text { weeks }\end{array}$ & 18 May 2017 \\
\hline \multirow{3}{*}{ PD-L1 } & Atezolizumab & Human IgG1k & Tecentriq & MPDL3280A, RG7446 & Genentech Inc. & $\begin{array}{l}1200 \mathrm{mg} \text {, every } \\
3 \text { weeks }\end{array}$ & 18 May 2016 \\
\hline & Durvalumab & Humanized IgG1k & Imfinzi & MEDI-4736 & $\begin{array}{l}\text { AstraZeneca } \\
\text { UK Limited }\end{array}$ & $\begin{array}{c}10 \mathrm{mg} / \mathrm{kg} \text {, every } \\
2 \text { weeks }\end{array}$ & 1 May 2017 \\
\hline & Avelumab & Human IgG1 & Bavencio & $\begin{array}{l}\text { MSB0010718C, } \\
\text { MSB0010682 }\end{array}$ & EMD Serono Inc. & $\begin{array}{c}10 \mathrm{mg} / \mathrm{kg} \text {, every } \\
2 \text { weeks }\end{array}$ & 9 May 2017 \\
\hline
\end{tabular}

All are for the patients with locally advanced or metastatic urothelial carcinoma and who has the prior platinum-based chemotherapy.

inhibitory mechanism and evade the host antitumor activity by suppressing the proliferation of immune cells, subsequently survive, and continue to proliferate.

Immune checkpoint inhibitors were developed to control immune escape tumors. The most widely studied immune checkpoint inhibitors are anti-CTLA-4, anti-PD-1, and anti-PD-L1 monoclonal antibodies [18-20] (Figure 1) which target the $\mathrm{T}$ cell regulatory pathways to augment antitumor immune responses [21]. These inhibitors have shown promising efficacy in melanoma [22], renal cell carcinoma [23], NSCLC [24, 25], and bladder UC [26]. As in the case with immunotherapy for other types of cancers, these drugs show limited response rate, but the efficacy in achieving longlasting benefits for some patients has changed the paradigm of cancer treatment. Due to the milestone discovery of the role of PD-1 [27], PD-L1 [28], and CTLA-4 [29] in inhibiting carcinogenesis, the 2017 Warren Alpert Foundation Prize was awarded to Drs. T. Honjo, L. Chen, James P. Allison, and colleagues to honor their significant contributions in the field of cancer immunotherapy [29]. This review focuses on the FDA-approved PD-1 and PD-L1 inhibitors in urothelial carcinoma.

\section{US Food and Drug Administration-Approved Immune Checkpoint Inhibitors in Urothelial Carcinoma}

The first checkpoint inhibitor approved for bladder UC was atezolizumab (Tecentriq) in 2016, which was the secondline therapy for patients who had received platinum-based chemotherapy. Response rates were around 15\% with median survival of 7.9 months [30-32]. Other approved drugs that followed atezolizumab include durvalumab (Imfinzi) [33-35], nivolumab (Opdivo) [36], and avelumab (Bavencio) [37], and the latest is pembrolizumab (Keytruda) $[38,39]$. All these US FDA-approved agents were approved for the treatment of patients with locally advanced or metastatic UC who experienced disease progression during or after platinum-based chemotherapy, or within 12 months of neoadjuvant or adjuvant treatment with platinum-based chemotherapy [30-42]. The clinical efficacy, adverse events, and recommended usage of these drugs are as follows.

3.1. Efficacy. Atezolizumab was the first immune checkpoint inhibitor approved by the US FDA on May 18, 2016, for the treatment of patients who experienced unsuccessful first-line platinum-based chemotherapy (Table 1). Atezolizumab is a human IgG1k antibody against the PD-L1 checkpoint. The US FDA-accelerated approval was obtained by the results of the IMvigor-210 study, a multicenter, single-arm trial of 310 patients with UC [30-32]. The IMvigor-210 study stratified patients with UC by PD-L1 expression levels in the tumor-infiltrating immune cells. Patients with $\geq 5 \%$ of tumor-infiltrating immune cells stained by PD-L1 in the tumor were categorized as a PD-L1-positive group. In this study, a total of $100(32 \%)$ and $210(68 \%)$ patients were categorized into PD-L1 positive and PD-L1 negative, respectively. The trial excluded patients with a history of autoimmune diseases or those who required systemic immunosuppressive medications. All patients received $1200 \mathrm{mg}$ of atezolizumab intravenously every 3 weeks. The efficacy was evaluated by Response Evaluation Criteria in Solid Tumors version 1.1 (RECIST v1.1). The objective response rate (ORR) of all patients was $14.8 \%$ (Table 2) [30, 31]. The median duration of response ranged from 2.1 to 13.8 months. The ORR for patients with PD-L1 positive versus those were negative was $26.0 \%$ and $9.5 \%$, respectively (Table 2 ) [30, 31].

Durvalumab is a humanized IgG1k antibody also against the PD-L1 checkpoint (Table 1) [33]. The US FDA granted accelerated approval to durvalumab for the treatment of patients with advanced or metastatic UC on May 1, 2017. The approval was based on a single-arm study of patients with UC who had unsuccessful first-line platinum-based chemotherapy $[33,34]$. Recently, the results from the durvalumab trial involving 191 patients with UC have been updated [35]. Durvalumab (10 $\mathrm{mg} / \mathrm{kg}$ every 2 weeks) was administered to patients intravenously. The efficacy was assessed using RECIST v1.1 criteria. The median duration of response ranged from 0.9 to 19.9 months. The trial also stratified patients with UC by PD-L1 expression levels. The ORR was $17.8 \%$ for all patients $(n=191)$ and was $27.6 \%(n=27)$ 


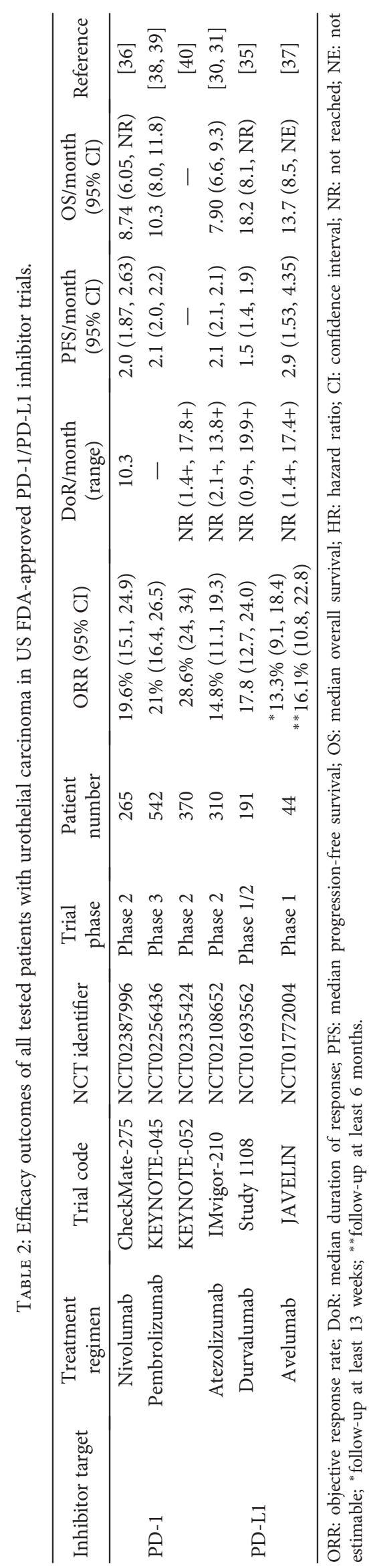


TABLE 3: Treatment-related adverse events of US FDA-approved PD-1/PD-L1 inhibitors in patients with urothelial carcinoma.

\begin{tabular}{|c|c|c|c|}
\hline Target & Inhibitor name & Treatment-related adverse events & Immune-related adverse events \\
\hline \multirow{2}{*}{ PD-1 } & Nivolumab & $\begin{array}{l}\text { Fatigue, decreased appetite, nausea, musculoskeletal } \\
\text { pain, diarrhea, rash }\end{array}$ & $\begin{array}{l}\text { Pneumonitis, hepatitis, colitis, endocrinopathies, } \\
\text { nephritis, renal dysfunction, encephalitis, rash }\end{array}$ \\
\hline & Pembrolizumab & $\begin{array}{l}\text { Fatigue, decreased appetite, nausea, musculoskeletal } \\
\text { pain, diarrhea, rash, pruritus, constipation }\end{array}$ & $\begin{array}{c}\text { Pneumonitis, hepatitis, colitis, endocrinopathies, } \\
\text { nephritis, renal dysfunction }\end{array}$ \\
\hline \multirow{3}{*}{ PD-L1 } & Atezolizumab & $\begin{array}{l}\text { Fatigue, decreased appetite, nausea, urinary tract } \\
\text { infection, pyrexia, constipation }\end{array}$ & $\begin{array}{c}\text { Pneumonitis, hepatitis, colitis, endocrinopathies } \\
\text { (thyroid disease, adrenal insufficiency, hypophysitis, } \\
\text { type } 1 \text { diabetes), meningitis/encephalitis, pancreatitis, } \\
\text { dermatitis/rash }\end{array}$ \\
\hline & Durvalumab & $\begin{array}{c}\text { Fatigue, decreased appetite, nausea, urinary tract } \\
\text { infection, diarrhea, musculoskeletal pain, constipation, } \\
\text { peripheral edema }\end{array}$ & $\begin{array}{l}\text { Pneumonitis, hepatitis, colitis, endocrinopathies } \\
\text { (thyroid disease, adrenal insufficiency, hypophysitis, } \\
\text { type } 1 \text { diabetes), nephritis }\end{array}$ \\
\hline & Avelumab & $\begin{array}{l}\text { Fatigue, decreased appetite, nausea, urinary tract } \\
\text { infection, musculoskeletal pain }\end{array}$ & $\begin{array}{l}\text { Pneumonitis, hepatitis, colitis, endocrinopathies, } \\
\text { nephritis, renal dysfunction }\end{array}$ \\
\hline
\end{tabular}

and $5.1 \%(n=4)$ in PD-L1 high expression and low (or negative) expression groups, respectively. The median OS was 18.2 months for all patients and was 20.0 months and 8.1 months in PD-L1 high expression and low (or negative) expression groups, respectively (Table 2) [35].

Avelumab is a human IgG1 antibody against the PDL1 checkpoint. Avelumab received US FDA-accelerated approval on May 9, 2017, based on the results of the openlabel, single-arm, multicenter JAVELIN study (Table 1) [37]. Avelumab was approved for the treatment of patients with UC who had disease progression after first-line platinum-based chemotherapy. In the JAVELIN trial, patients received avelumab $(10 \mathrm{mg} / \mathrm{kg}$ every 2 weeks $)$ intravenously until disease progression or intolerable toxicity. Before avelumab administration, all patients received antihistamine and acetaminophen. The ORRs at 13-week $(n=30)$ and 6-month $(n=26)$ follow-ups were $13.3 \%$ and $16.1 \%$, respectively. The median duration of response ranged from 1.4 to 17.4 months (Table 2) [37].

Nivolumab is a human IgG4 antibody against the PD-1 checkpoint. Based on a single-arm clinical study, CheckMate-275 [36], the US FDA granted accelerated approval to nivolumab on February 2, 2017, for the treatment of UC after unsuccessful first-line platinum-based chemotherapy (Table 1). Nivolumab was also the first immune checkpoint inhibitor approved in the European Union for UC treatment on June 4, 2017. In the CheckMate-275 trial, nivolumab was administered to 270 patients with UC ( $3 \mathrm{mg} / \mathrm{kg}$ every 2 weeks) until disease progression or intolerable toxicity. The ORR following RECIST criteria was $19.6 \%$. Seven patients $(2.6 \%)$ had complete responses, whereas 46 (17\%) had a partial response. The median duration of response was 10.3 months, and the median overall survival (OS) was 8.7 months (Table 2) [36].

Pembrolizumab is a humanized IgG4 antibody against the PD-1 checkpoint. Pembrolizumab is the latest immune checkpoint inhibitor approved by the US FDA on May 18, 2017, for the treatment of patients with UC (Table 1). In addition to the approval of second-line indication, pembrolizumab also received US FDA-accelerated approval for firstline indication for UC treatment. The first- and second-line indications were approved based on KEYNOTE-052 [40] and KEYNOTE-045 [38, 39] trials, respectively. In the KEYNOTE-052 trial, 370 patients with UC who were not eligible for cisplatin-based chemotherapy were enrolled and administered with pembrolizumab (200 mg every 3 weeks). The median follow-up was 7.8 months, and the ORR was $28.6 \%$. The median duration of response ranged from 1.4 to 17.8 months. In the KEYNOTE-045 trial, 542 patients with UC were randomly assigned to receive either pembrolizumab (200 mg every 3 weeks; $n=270$ ) or the investigator's choice of a chemotherapy regimen (every 3 weeks, $n=272$ ) [38]. This trial produced significant improvements in the median OS and ORRs in both pembrolizumab- and chemotherapytreated groups. The median OS was 10.3 and 7.4 months in pembrolizumab- and chemotherapy-treated groups, respectively (hazard ratio: $0.73 ; 95 \% \mathrm{CI}: 0.59-0.91 ; p=0.004$ ). The ORRs were $21 \%$ and $11 \%$ for pembrolizumab- and chemotherapy-treated groups, respectively $(p=0.002)$. However, no significant differences were observed in the progression-free survival between the two regimen groups (Table 2) [38, 39].

3.2. Adverse Events. Table 3 presents the adverse events of the five US FDA-approved PD-1/PD-L1 inhibitors for patients with UC [30-38, 40, 43-47]. The most common treatmentrelated adverse events observed in about $15-20 \%$ of treated patients include fatigue, decreased appetite, nausea, and musculoskeletal pain. Urinary tract infection was reported in patients treated with the three PD-L1 inhibitors. Constipation was observed in the atezolizumab-, durvalumab-, and pembrolizumab-treated groups. In addition, pyrexia and peripheral edema were reported in the atezolizumaband durvalumab-treated groups, respectively. Furthermore, the pembrolizumab-treated group had pruritus and rash. Diarrhea is commonly seen in PD-L1- and durvalumabtreated patients.

In addition, immune-targeted agents that can cause dysimmune toxicities in any tissue but mainly affect the lung, liver, gut, endocrine glands, and skin caused immunerelated adverse events (IRAEs) [48]. Although severe IRAEs are rare, once occurred, they can be life-threatening if 
TABLE 4: Selected new or ongoing clinical trials of PD-1/PD-L1 inhibitors for the treatment of urothelial carcinoma.

\begin{tabular}{lcccc}
\hline NCT identifier & Interventions & Recruitment & Phases & Locations \\
\hline NCT03113266 & Anti-PD-1 monoclonal antibody & Recruiting & Phase 2 & China \\
NCT03287050 & Pembrolizumab/radiation & Not yet recruiting & Early phase 1 & United States \\
NCT03240016 & Pembrolizumab/abraxane & Not yet recruiting & Phase 2 & United States \\
NCT02807636 & Atezolizumab/carboplatin/gemcitabine/cisplatin/placebo & Recruiting & Phase 3 & Globe \\
NCT02853305 & Pembrolizumab/cisplatin/carboplatin/gemcitabine & Recruiting & Phase 3 Globe \\
NCT03219775 & Nivolumab/ipilimumab & Recruiting & Phase 2 & Germany \\
NCT02500121 & Pembrolizumab/placebo & Recruiting & Phase 2 & United States \\
NCT02450331 & Atezolizumab & Recruiting & Phase 3 & Globe \\
NCT03115801 & Atezolizumab/radiation & Recruiting & Phase 2 & United States \\
NCT03244384 & Pembrolizumab/clinical observation/biomarker analysis & Recruiting & United States \\
NCT02451423 & Atezolizumab dose level 1/dose level 2/dose level 3 & Recruiting & Phase 2 & United States \\
NCT02897765 & NEO-PV-01/nivolumab/adjuvant & Recruiting & Phase 1 & United States \\
NCT02845323 & Nivolumab + urelumab/nivolumab monotherapy & Recruiting & Phase 2 & United States \\
NCT02736266 & Pembrolizumab & Recruiting & Phase 2 & Italy \\
NCT03237780 & Atezolizumab/eribulin mesylate/biomarker analysis & Not yet recruiting & Phase 2 & United States \\
\hline
\end{tabular}

managed inappropriately [49]. Table 3 lists the common IRAEs of checkpoint inhibitor-treated patients. All five checkpoint inhibitor-treated groups might have pneumonitis, hepatitis, colitis, and endocrinopathies (e.g., thyroid disease, adrenal insufficiency, hypophysitis, and type 1 diabetes). Nephritis and renal dysfunction were commonly observed in all drug-treated groups except the atezolizumab-treated group. Meningitis/encephalitis and dermatitis/rash were observed in the atezolizumab- and nivolumab-treated groups. Pancreatitis may also in the atezolizumab-treated group. Other details of the IRAEs caused by checkpoint inhibitors are described elsewhere [6, 43-47].

3.3. Recommended Usage. Table 1 presents the recommended usage of the US FDA-approved immune checkpoint inhibitors for UC treatment. These antibodies are administered intravenously. The recommended doses and schedules for atezolizumab, nivolumab, durvalumab, avelumab, and pembrolizumab are $1200 \mathrm{mg}$ every 3 weeks, $240 \mathrm{mg}$ every 2 weeks, $10 \mathrm{mg} / \mathrm{kg}$ every 2 weeks, $10 \mathrm{mg} / \mathrm{kg}$ over a 60 -minute influx every 2 weeks, and $200 \mathrm{mg}$ over a 30-minute influx every 3 weeks, respectively, until disease progression or intolerable toxicity [30-40].

\section{Discussion}

Although upper tract urothelial carcinoma (UTUC) was identified with molecular profiling approaches that were different from those for bladder UC [50], the immune checkpoint inhibitors performed with promising efficacy in both UTUC and bladder UC [51]. However, many concerns remain. For example, the exact mechanism underlying the dominant role of PD-L1 expression in the efficacy of anti-PD-1/PD-L1 antibodies remains unclear. Furthermore, the influence of patients' genetic backgrounds, particularly racial differences, warrants further investigation.
According to our review of the relevant literature, previous studies did not provide the nucleotide sequence or protein compositions of PD-1/PD-L1 immune checkpoints in patients with UC. The relationships between the antigenbinding sites (paratopes) on the therapeutic monoclonal antibody inhibitors and the antibody-binding sites (epitopes) on the checkpoint proteins remain unclear. Nonetheless, UC has been identified as the tumor with high and heterogeneous mutation burden [52]. The genetic characteristics affect the efficacy of anticancer agents. The observation on tyrosine kinase inhibitor (TKI) treatments for NSCLC demonstrated a paradigm shift on the associations between mutation type and drug efficacy; moreover, even a single site mutation could have a substantial influence on drug sensitivity or resistance [53]. There is an urgent need to identify a biomarker as a clinical outcome predictor for patients with UC who can benefit from the anti-PD-1/PD-L1 immunotherapy.

Currently, PD-L1 is regarded as a biomarker in PD-1/PDL1 inhibitor trials $[20,54-56]$ trials although the exact role of PD-L1 expression in the therapeutic efficacy of PD-1/PD-L1 inhibitors remains controversial [57]. For clinical practice, PD-L1 expression level of patients with metastatic melanoma or NSCLC is typically examined to determine whether the patients are suitable for treatment of anti-PD-1/PD-L1 immunotherapy [58]. For patients with UC, VENTANA PD-L1 SP142 and SP263 assays were used to classify them into PD-L1-positive or PD-L1-negative cohorts in atezolizumab and durvalumab trials, respectively [30, 31, 33, 35]. Those trials indicated patients with higher PD-L1 expression exhibiting improved efficacy compared to those with lower PD-L1 expression. However, of the variations in techniques, platforms, diverse specimens, tumor and immune microenvironment and the positive cutoff of PD-L1 expression complicate the standardization of decision-making in clinical applications [57]. Therefore, the classification of PD-L1positive and PD-L1-negative groups for cancer patients is usually defined dynamically based on different assays or 
cutoffs. Currently, we suggest using PD-L1 expression level for outcome assessments but not for patient selections. Hence, the optimization of biomarker assays to identify the ideal population for anti-PD-1/PD-L1 immunotherapy is crucial for clinical practice $[57,58]$. Alternatively, stratifying patients with UC based on the epitope sequences of their checkpoints and then applying the subtypes of the epitopes to develop the corresponding anti-PD-1/PD-L1 antibodies may contribute to the optimization of personalized and precision medicine.

Additionally, these PD-1/PD-L1 inhibitors may exert synergistic effects with other anticancer agents to prolong patients' survival or reduce side effects. Table 4 shows selected new or ongoing clinical studies of PD-1/PD-L1 inhibitors for the treatment of UC. Those interventions are monotherapy of PD-1/PD-L1 inhibitors or combination therapy with anti-CTLA-4 antibodies, chemotherapy agents, or radiotherapy. Some studies are designed to discover the relationships between biomarker and the efficacy of PD-1/PD-L1 inhibitors as well as the effect of difference dosage levels. Their results may provide new clues or strategies in winning the fight against UC in the future.

In this compact but comprehensive review, we summarized the background information of the five US FDA approved PD-1 and PD-L1 checkpoint inhibitors as well as elucidate their mechanism of actions (MOA). We outlined their drug efficacy, safety, and adverse events from the clinical trials of patients with UC. These therapeutic antibodies have shown promising results in their respective FDAapproved trials and have given new hope to those who are suffering from advanced or metastatic UC. Further largescale clinical trials of checkpoint inhibitor will reveal the optimal administration of these drugs and allow more patients with UC to benefit from immunotherapy treatments.

\section{Conflicts of Interest}

The authors declare that they have no financial or commercial conflicts of interest related to the subject matter or materials discussed in the manuscript.

\section{Acknowledgments}

This review article was partially supported by Taiwan Maple Urological Association. The authors thank Chun-I Pan for the illustration of the figure. This article has been proofread by An-Chi Chen (Tamkang University, Taiwan).

\section{References}

[1] O. Sanli, J. Dobruch, M. A. Knowles et al., "Bladder cancer," Nature Reviews Disease Primers, vol. 3, article 17022, 2017.

[2] R. L. Siegel, K. D. Miller, and A. Jemal, "Cancer statistics, 2017," CA: a Cancer Journal for Clinicians, vol. 67, no. 1, pp. 7-30, 2017.

[3] J. C. Park and N. M. Hahn, "Emerging role of immunotherapy in urothelial carcinoma-future directions and novel therapies," Urologic Oncology, vol. 34, no. 12, pp. 566-576, 2016.
[4] S. A. Hussain and N. D. James, "The systemic treatment of advanced and metastatic bladder cancer," Lancet Oncology, vol. 4, no. 8, pp. 489-497, 2003.

[5] J. J. Kim, "Recent advances in treatment of advanced urothelial carcinoma," Current Urology Reports, vol. 13, no. 2, pp. 147152,2012

[6] D. P. Petrylak, "Immunotherapy: the wave of the future in bladder cancer?," Clinical Genitourinary Cancer, vol. 15, no. 3, pp. S3-S17, 2017.

[7] B. J. Roth and D. F. Bajorin, "Advanced bladder cancer: the need to identify new agents in the post-M-VAC (methotrexate, vinblastine, doxorubicin and cisplatin) world," The Journal of Urology, vol. 153, no. 3, pp. 894-900, 1995.

[8] J. D. Ramos and E. Y. Yu, "Making urothelial carcinomas less immune to immunotherapy," Urologic Oncology, vol. 34, no. 12 , pp. 534-537, 2016.

[9] J. García-Donas, A. Font, B. Pérez-Valderrama et al., "Maintenance therapy with vinflunine plus best supportive care versus best supportive care alone in patients with advanced urothelial carcinoma with a response after first-line chemotherapy (MAJA; SOGUG 2011/02): a multicentre, randomised, controlled, open-label, phase 2 trial," Lancet Oncology, vol. 18, no. 5, pp. 672-681, 2017.

[10] S. Gupta, D. Gill, A. Poole, and N. Agarwal, "Systemic immunotherapy for urothelial cancer: current trends and future directions," Cancers, vol. 9, no. 2, article E15, 2017.

[11] G. Sonpavde, B. S. Jones, J. Bellmunt, T. K. Choueiri, and C. N. Sternberg, "Future directions and targeted therapies in bladder cancer," Hematology/Oncology Clinics of North America, vol. 29, no. 2, pp. 361-376, 2015.

[12] S. Wang, Y. Song, and D. Liu, "EAI045: the fourth-generation EGFR inhibitor overcoming T790M and C797S resistance," Cancer Letters, vol. 385, pp. 51-54, 2017.

[13] M. Friese-Hamim, F. Bladt, G. Locatelli, U. Stammberger, and A. Blaukat, "The selective c-Met inhibitor tepotinib can overcome epidermal growth factor receptor inhibitor resistance mediated by aberrant c-Met activation in NSCLC models," American Journal of Cancer Research, vol. 7, no. 4, pp. 962-972, 2017.

[14] T. Kurata, A. Nakaya, T. Yokoi et al., "Phase I/II study of erlotinib, carboplatin, pemetrexed, and bevacizumab in chemotherapy-naïve patients with advanced non-squamous non-small cell lung cancer harboring epidermal growth factor receptor mutation," Genes and Cancer, vol. 8, no. 5-6, pp. 559$565,2017$.

[15] H. R. Kourie, E. El Rassy, F. Clatot, E. de Azambuja, and M. Lambertini, "Emerging treatments for HER2-positive early-stage breast cancer: focus on neratinib," OncoTargets and Therapy, vol. 10, pp. 3363-3372, 2017.

[16] C. Chung and M. S. Lam, "Pertuzumab for the treatment of human epidermal growth factor receptor type 2-positive metastatic breast cancer," American Journal of Health-System Pharmacy, vol. 70, no. 18, pp. 1579-1587, 2013.

[17] M. Miura, "Therapeutic drug monitoring of imatinib, nilotinib, and dasatinib for patients with chronic myeloid leukemia," Biological \& Pharmaceutical Bulletin, vol. 38, no. 5, pp. 645-654, 2015.

[18] E. I. Buchbinder and A. Desai, "CTLA-4 and PD-1 pathways: similarities, differences, and implications of their inhibition," American Journal of Clinical Oncology, vol. 39, no. 1, pp. 98-106, 2016. 
[19] J. Wang, R. Yuan, W. Song, J. Sun, D. Liu, and Z. Li, "PD-1, PD-L1 (B7-H1) and tumor-site immune modulation therapy: the historical perspective," Journal of Hematology \& Oncology, vol. 10, no. 1, p. 34, 2017.

[20] W. Ma, B. M. Gilligan, J. Yuan, and T. Li, "Current status and perspectives in translational biomarker research for $\mathrm{PD}-1 / \mathrm{PD}$ L1 immune checkpoint blockade therapy," Journal of Hematology \& Oncology, vol. 9, no. 1, p. 47, 2016.

[21] P. Sharma and J. P. Allison, "The future of immune checkpoint therapy," Science, vol. 348, no. 6230, pp. 56-61, 2015.

[22] M. Sanlorenzo, I. Vujic, C. Posch et al., "Melanoma immunotherapy," Cancer Biology \& Therapy, vol. 15, no. 6, pp. 665674, 2014.

[23] M. C. Ornstein and B. I. Rini, "The safety and efficacy of nivolumab for the treatment of advanced renal cell carcinoma," Expert Review of Anticancer Therapy, vol. 16, no. 6, pp. 577$584,2016$.

[24] S. L. Topalian, F. S. Hodi, J. R. Brahmer et al., "Safety, activity, and immune correlates of anti-PD-1 antibody in cancer," New England Journal of Medicine, vol. 366, no. 26, pp. 2443-2454, 2012.

[25] J. R. Brahmer, S. S. Tykodi, L. Q. Chow et al., "Safety and activity of anti-PD-L1 antibody in patients with advanced cancer," New England Journal of Medicine, vol. 366, no. 26, pp. 24552465, 2012.

[26] T. Powles, J. P. Eder, G. D. Fine et al., "MPDL3280A (anti-PDL1) treatment leads to clinical activity in metastatic bladder cancer," Nature, vol. 515, no. 7528, pp. 558-562, 2014.

[27] Y. Ishida, Y. Agata, K. Shibahara, and J. Honjo, "Induced expression of PD-1, a novel member of the immunoglobulin gene superfamily, upon programmed cell death," The EMBO Journal, vol. 11, no. 11, pp. 3887-3895, 1992.

[28] H. Dong, G. Zhu, K. Tamada, and L. Chen, "B7-H1, a third member of the B7 family, co-stimulates T-cell proliferation and interleukin-10 secretion," Nature Medicine, vol. 5, no. 12, pp. 1365-1369, 1999.

[29] "2017 Warren Alpert Foundation Prize Recipients Announced," https://warrenalpert.org/news/2017-warrenalpert-foundation-prize-recipients-announced.

[30] J. E. Rosenberg, J. Hoffman-Censits, T. Powles et al., "Atezolizumab in patients with locally advanced and metastatic urothelial carcinoma who have progressed following treatment with platinum-based chemotherapy: a single-arm, multicentre, phase 2 trial," Lancet, vol. 387, no. 10031, pp. 1909-1920, 2016.

[31] Y. M. Ning, D. Suzman, V. E. Maher et al., "FDA approval summary: atezolizumab for the treatment of patients with progressive advanced urothelial carcinoma after platinumcontaining chemotherapy," The Oncologist, vol. 22, no. 6, pp. 743-749, 2017.

[32] A. Markham, "Atezolizumab: first global approval," Drugs, vol. 76, no. 12, pp. 1227-1232, 2016.

[33] C. Massard, M. S. Gordon, S. Sharma et al., "Safety and efficacy of durvalumab (MEDI4736), an anti-programmed cell death ligand-1 immune checkpoint inhibitor, in patients with advanced urothelial bladder cancer," Journal of Clinical Oncology, vol. 34, no. 26, pp. 3119-3125, 2016.

[34] V. Brower, "Anti-PD-L1 inhibitor durvalumab in bladder cancer," Lancet Oncology, vol. 17, no. 7, article e275, 2016.

[35] T. Powles, P. H. O'Donnell, C. Massard et al., "Efficacy and safety of durvalumab in locally advanced or metastatic urothelial carcinoma: updated results from a phase $1 / 2$ openlabel study," JAMA Oncology, vol. 3, no. 9, article e172411, 2017.

[36] P. Sharma, M. Retz, A. Siefker-Radtke et al., "Nivolumab in metastatic urothelial carcinoma after platinum therapy (CheckMate 275): a multicentre, single-arm, phase 2 trial," Lancet Oncology, vol. 18, no. 3, pp. 312-322, 2017.

[37] C. R. Heery, G. O'Sullivan-Coyne, R. A. Madan et al., "Avelumab for metastatic or locally advanced previously treated solid tumours (JAVELIN solid tumor): a phase 1a, multicohort, dose-escalation trial," Lancet Oncology, vol. 18, no. 5, pp. 587-598, 2017.

[38] J. Bellmunt, R. de Wit, D. J. Vaughn et al., "Pembrolizumab as second-line therapy for advanced urothelial carcinoma," New England Journal of Medicine, vol. 376, no. 11, pp. 1015-1026, 2017.

[39] J. M. Lavoie, S. Bidnur, P. C. Black, and B. J. Eigl, "Expanding immunotherapy options for bladder cancer," Urology, vol. 106, no. 9, pp. 1-2, 2017.

[40] A. Balar, J. Bellmunt, P. H. O’Donnell et al., “Pembrolizumab (pembro) as first-line therapy for advanced/unresectable or metastatic urothelial cancer: preliminary results from the phase 2 KEYNOTE-052 study," Annals of Oncology, vol. 27, Supplement_6, 2016.

[41] C. Zichi, M. Tucci, G. Leone et al., "Immunotherapy for patients with advanced urothelial cancer: current evidence and future perspectives," BioMed Research International, vol. 2017, p. 5618174, 2017.

[42] M. S. Farina, K. T. Lundgren, and J. Bellmunt, "Immunotherapy in urothelial cancer: recent results and future perspectives," Drugs, vol. 77, no. 10, pp. 1077-1089, 2017.

[43] "FDA Highlights of Prescribing Information: Atezolizumab," https://www.accessdata.fda.gov/drugsatfda_docs/label/2016/7 61034s000lbl.pdf.

[44] "FDA Highlights of Prescribing Information: Durvalumab," https://www.accessdata.fda.gov/drugsatfda_docs/label/2017/7 61069s000lbl.pdf.

[45] "FDA Highlights of Prescribing Information: Avelumab," https://www.accessdata.fda.gov/drugsatfda_docs/label/2017/7 61078s000lbl.pdf.

[46] "FDA Highlights of Prescribing Information: Nivolumab," https://www.accessdata.fda.gov/drugsatfda_docs/label/2017/1 25554s024lbl.pdf.

[47] "FDA Highlights of Prescribing Information: Pembrolizumab," https://www.accessdata.fda.gov/drugsatfda_docs/label/ 2017/125514s017s018lbl.pdf.

[48] J. M. Michot, C. Bigenwald, S. Champiat et al., "Immunerelated adverse events with immune checkpoint blockade: a comprehensive review," European Journal of Cancer, vol. 54, pp. 139-148, 2016.

[49] S. Champiat, O. Lambotte, E. Barreau et al., "Management of immune checkpoint blockade dysimmune toxicities: a collaborative position paper," Annals of Oncology, vol. 27, no. 4, pp. 559-574, 2016.

[50] R. Mathieu and S. F. Shariat, "Building bridges in urothelial carcinoma to face common challenges," Translational Andrology and Urology, vol. 5, no. 5, pp. 745-748, 2016.

[51] J. B. Aragon-Ching, "Challenges and advances in the diagnosis, biology, and treatment of urothelial upper tract and bladder carcinomas," Urologic Oncology, vol. 35, no. 7, pp. $462-464,2017$. 
[52] S. A. Mullane and J. Bellmunt, "Cancer immunotherapy: new applications in urologic oncology," Current Opinions in Urology, vol. 26, no. 6, pp. 556-563, 2016.

[53] S. Wang, S. Cang, and D. Liu, "Third-generation inhibitors targeting EGFR T790M mutation in advanced non-small cell lung cancer," Journal of Hematology \& Oncology, vol. 9, no. 1, p. $34,2016$.

[54] A. I. Daud, K. Loo, M. L. Pauli et al., "Tumor immune profiling predicts response to anti-PD-1 therapy in human melanoma," The Journal of Clinical Investigation, vol. 126, no. 9, pp. 3447-3452, 2016.

[55] M. Callea, L. Albiges, M. Gupta et al., "Differential expression of PD-L1 between primary and metastatic sites in clear-cell renal cell carcinoma," Cancer Immunology Research, vol. 3, no. 10, pp. 1158-1164, 2015.

[56] C. Y. Yang, M. W. Lin, Y. L. Chang, C. T. Wu, and P. C. Yang, "Programmed cell death-ligand 1 expression in surgically resected stage I pulmonary adenocarcinoma and its correlation with driver mutations and clinical outcomes," European Journal of Cancer, vol. 50, no. 7, pp. 1361-1369, 2014.

[57] D. Liu, S. Wang, and W. Bindeman, "Clinical applications of PD-L1 bioassays for cancer immunotherapy," Journal of Hematology \& Oncology, vol. 10, no. 1, p. 110, 2017.

[58] L. P. Diggs and E. C. Hsueh, "Utility of PD-L1 immunohistochemistry assays for predicting PD-1/PD-L1 inhibitor response," Biomarker Research, vol. 5, no. 1, p. 12, 2017. 


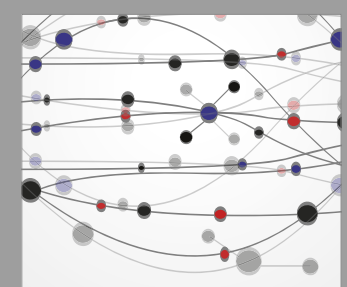

The Scientific World Journal
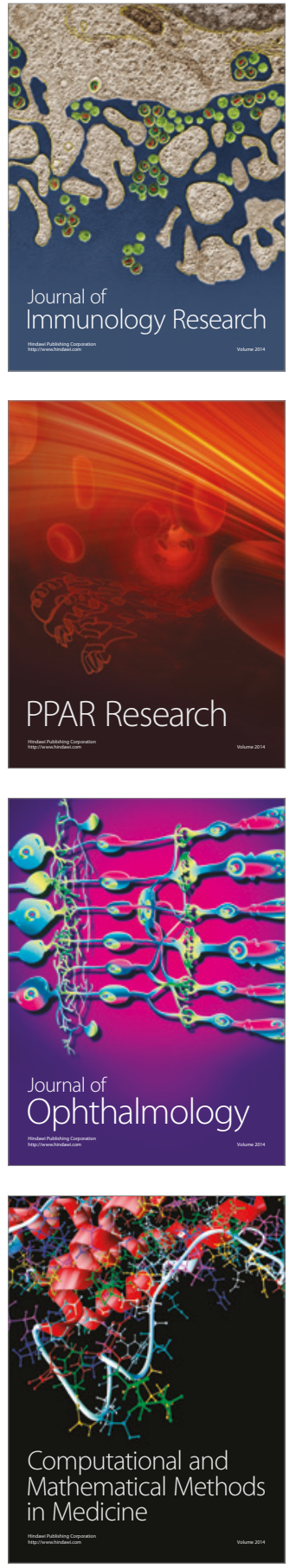

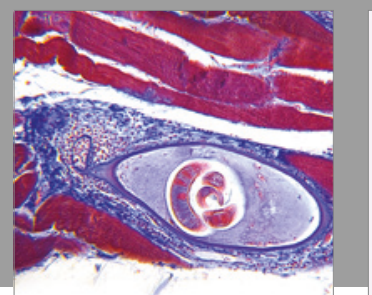

Gastroenterology Research and Practice
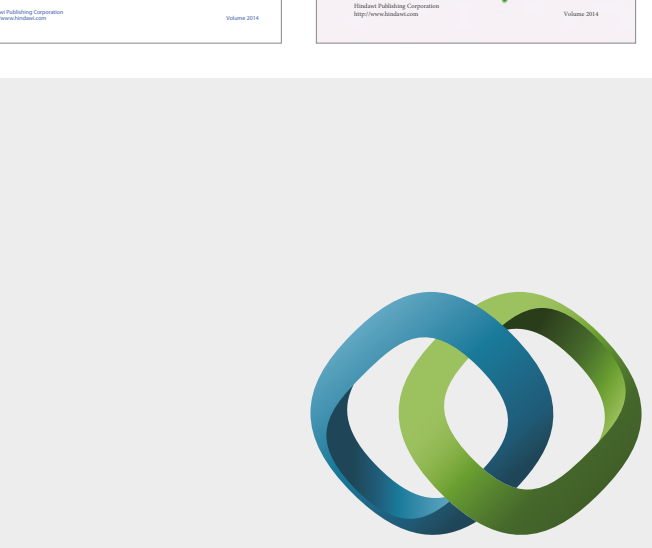

\section{Hindawi}

Submit your manuscripts at

https://www.hindawi.com
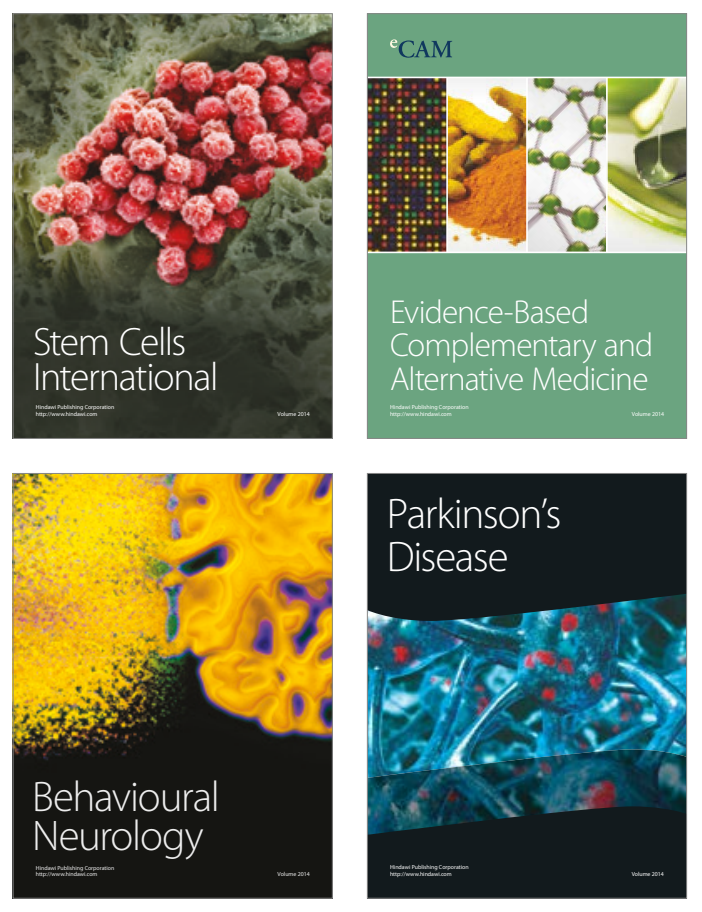
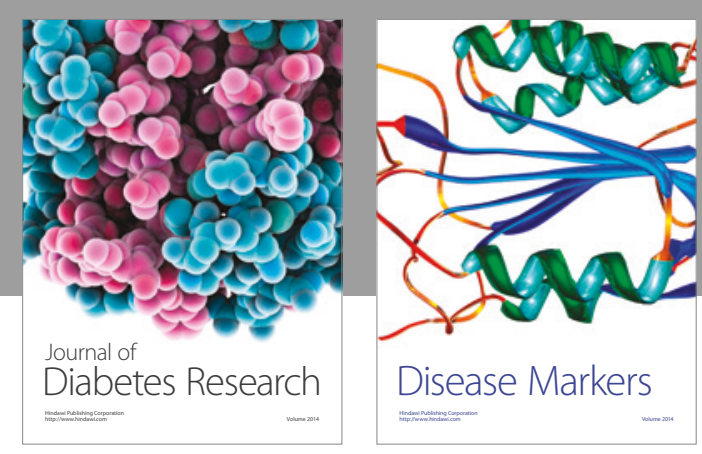

Disease Markers
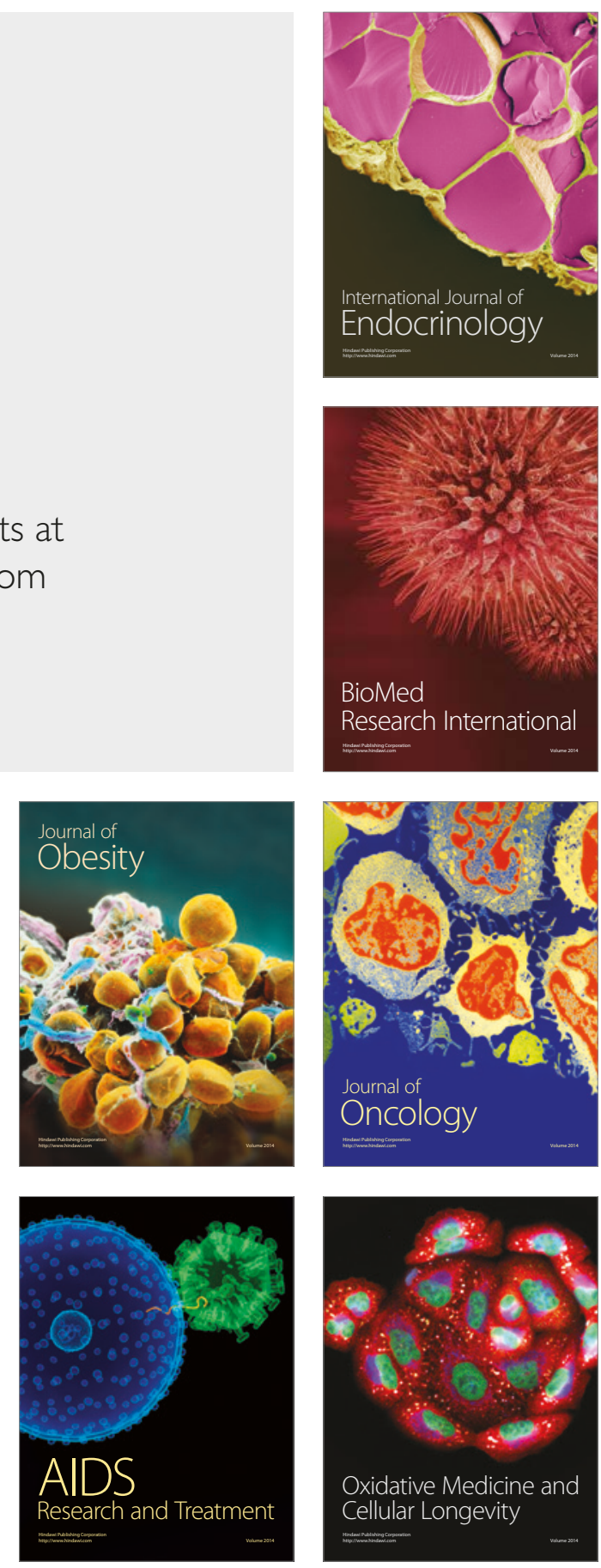